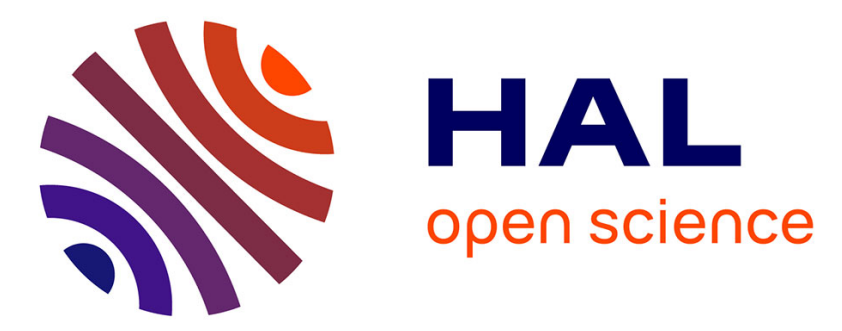

\title{
Controlling surface morphology by nanocrystalline/amorphous competitive self-phase separation in thin films: Thickness-modulated reflectance and interference phenomena
}

A. Borroto, S. Bruyère, S. Migot, J.F. Pierson, T. Gries, F. Mücklich, D. Horwat

\section{To cite this version:}

A. Borroto, S. Bruyère, S. Migot, J.F. Pierson, T. Gries, et al.. Controlling surface morphology by nanocrystalline/amorphous competitive self-phase separation in thin films: Thicknessmodulated reflectance and interference phenomena. Acta Materialia, 2019, 181, pp.78-86. 10.1016/j.actamat.2019.09.036 . hal-02326746

\section{HAL Id: hal-02326746 \\ https://hal.science/hal-02326746}

Submitted on 6 Jul 2021

HAL is a multi-disciplinary open access archive for the deposit and dissemination of scientific research documents, whether they are published or not. The documents may come from teaching and research institutions in France or abroad, or from public or private research centers.
L'archive ouverte pluridisciplinaire HAL, est destinée au dépôt et à la diffusion de documents scientifiques de niveau recherche, publiés ou non, émanant des établissements d'enseignement et de recherche français ou étrangers, des laboratoires publics ou privés. 


\title{
Controlling surface morphology by nanocrystalline/amorphous competitive self-phase separation in thin films: thickness-modulated reflectance and interference phenomena
}

\author{
A. Borroto ${ }^{\mathrm{a}, \mathrm{b}}$, S. Bruyère ${ }^{\mathrm{a}}$, S. Migot ${ }^{\mathrm{a}}$, J.F. Pierson ${ }^{\mathrm{a}}$, T. Gries $^{\mathrm{a}}$, F. Mücklich ${ }^{\mathrm{b}}$, D. Horwat ${ }^{\mathrm{a}, *}$ \\ ${ }^{a}$ Institut Jean Lamour (UMR CNRS 7198), Université de Lorraine, Campus ARTEM, 54000 Nancy, France. \\ ${ }^{b}$ Department of Materials Science and Engineering, Chair of Functional Materials, Saarland University, Campus D3.3, D-66123 Saarbrücken, Germany.
}

\begin{abstract}
Controlling surface morphology is a key issue for obtaining functional materials with surface-based properties. In this paper, we explore the possibility of using the self-separation of phases as a way of controlling the surface morphology features. We demonstrate using X-ray diffraction and transmission electron microscopy that a competitive self-separation of a nanocrystalline and an amorphous phases occurs in co-sputtered $\mathrm{Zr}$-Mo thin films with a Mo content of 60 at\%, corresponding to a composition intermediate to those necessary to form single-phased amorphous and nanocrystalline films. The dependence of the residual stress with the thickness at the biphased composition is discussed in terms of the morphology evolution and a possible mechanism for the self-separation of phases is presented. We show that the self-separation of phases as presented here is not limited to $\mathrm{Zr}$-Mo alloys and can be extended to other systems. By changing the film thickness, it is possible to change the surface morphology of the films at the biphasic composition, due to the competitive growth of the nanocrystalline phase in the amorphous phase. In this way, it was possible to control the surface roughness and, because of this, tuning the film reflectance at a determined wavelength. The occurrence of an interference pattern in the reflectance spectra was discussed and associated to the presence of two different height levels at the film surface.
\end{abstract}

Keywords:

\section{Introduction}

The fabrication of new functional surfaces plays an important role in the development of the modern industry. Many efforts have been made in order to modify and control the surface morphology of a variety of materials by using different techniques $[1,2,3,4]$. The functionalities of surface-modified materials are wide: controllable wettability $[5,6]$, biomedical applications [7], solar cells [8], among others. Meanwhile, phase separation phenomenon is widely observed in thin films and has been thoroughly studied in order to manipulate resulting functional properties. Different configurations could be obtained, such as the nanodispersion of metal or ceramic particles in a ceramic matrix $[9,10]$. Alternatively, thin films showing lateral self-separation of ceramic phases could be obtained in case of specific epitaxial relationships between phases or between at least one phase and the substrate $[11,12,13]$. Apart for some specific cases (see for instance $[14,15]$ ), the topography of the film surface has scarcely been reported to be markedly affected by the phase separation. To the best of our knowledge, surface morphology modification induced by phase separation has not been exploited so far to manipulate the functional properties.

In recent years, Zr-rich Zr-Mo alloys obtained by different metallurgical methods have been investigated for biomedical application $[16,17,18,19,20]$. They have proven to exhibit

\footnotetext{
${ }^{*}$ Corresponding author

Email address: david.horwat@univ-lorraine.fr (D. Horwat)
}

low magnetic susceptibility that made this system a potential candidate for medical devices used under magnetic resonance imaging (MRI)[16, 17]. In their thin films form, Zr-Mo alloys have been obtained by magnetron sputtering in a wide range of compositions, exhibiting improved corrosion resistance [21] and mechanical properties [22] with respect to those of bulk $\mathrm{Zr}$ and Mo. Such difference results from the specific microstructure obtained in thin film form compared to the bulk state. Effectively, the cooling rates associated to the rapid transition from the vapor to the solid phase in sputter-deposited metallic alloys can be high enough to disable the crystallization of many systems. This fact can be used for the formation of amorphous and nanocrystalline structures and to tailor the properties of so-produced metallic alloy films. This is the case for sputterdeposited Zr-Mo thin films where a structural transition from an amorphous to a nanocrystalline phase was found around 60 at $\%$ Mo [21, 22]. Yet, the nature of the transition has not been addressed. It appeared as a singularity in the optical properties within the explored compositions in sputtered $\mathrm{Zr}$-Mo thin film [22]. A similar situation was observed in $\mathrm{Zr}-\mathrm{W}$ thin films [23] and, as will be shown, in the case of Ti-O films, suggesting that a general process could be in action, detectable only with very precise variation of the chemical composition.

In this paper, we demonstrate that a competitive selfseparation of an amorphous phase and a nanocrystalline phase occurs for Zr-Mo thin films obtained at a composition intermediate (around 60 at\% Mo) to those necessary to form singlephased amorphous and nanocrystalline films. Residual stress 
measurements, often used on single metallic elements, are used here for explaining the dynamical growth with thickness of Zr-Mo films obtained at the biphased composition, proving the usefulness of this technique when studying more complex microstructures. Moreover, we show that, because of the phase separation, the surface morphology can be modulated. In this way, the surface roughness, largely known to influence light scattering [24, 25, 26], can be tuned and, consequently, a thickness-controlled reflectance is achieved. It is important to remark that the competitive phase separation and its consequence in the modulation of the surface morphology can be extended to other systems.

\section{Experimental details}

Zr-Mo thin films were deposited on (100) silicon single crystal substrates by DC magnetron co-sputtering of $\mathrm{Zr}$ and $\mathrm{Mo}$ metallic targets in the presence of argon. The sputtering chamber was pumped down via a mechanical and a turbo-molecular pump allowing a base vacuum of $10^{-6} \mathrm{~Pa}$. The cathodes were mounted in a confocal configuration. The targets to substrate distance was fixed at $9 \mathrm{~cm}$. The argon flow rate was fixed at $30 \mathrm{sccm}$ and a working argon pressure of $2 \mathrm{~Pa}$ was used. To ensure the homogeneity of the films, all the depositions were made with the substrate holder in rotating mode. The zirconium target $(50.8 \mathrm{~mm}$ diameter, $3 \mathrm{~mm}$ thick and purity higher than 99.9\%) was powered by an Maxim 1000 DC generator using a discharge current of $0.3 \mathrm{~A}$. The molybdenum target $(50.8 \mathrm{~mm}$ in diameter, 3 mm-thick and purity higher than 99.9\%) was powered by an Maxim 1000 DC generator using discharge currents of $0.2,0.28$ and 0.4 A, depending of the desired Mo content. The films were deposited without external heating. The selfestablished temperature at the substrate was measured using a thermocouple and did not exceed $45^{\circ} \mathrm{C}$ during growth.

$\mathrm{X}$-ray diffraction (XRD) measurements were conducted in the Bragg-Brentano configuration with $\mathrm{K}_{\alpha} \mathrm{Cu}$ radiation $(\lambda=$ $1.5406 \AA$ ) using an AXS Bruker D8 Advance diffractometer. To analyze the morphology of the samples, top and crosssectional scanning electron micrographs were taken in a Philips XL-30 S-FEG scanning electron microscope (SEM). Crosssection SEM samples of films were prepared by breaking the film after scratching the back side with a diamond tip. The film thicknesses were measured using the cross-sectional scanning electron micrographs. Transmission electron microscopy (TEM) investigations were performed using a cold FEG JEOL ARM200 microscope. For this analysis, cross-section TEM samples of films were prepared using a focused ion beam (FIB)scanning electron microscope (SEM) dual beam system (FEI Helios 600). The films topography and RMS roughness were examined in resonant mode using a Nano-Observer Atomic Force Microscope developed by CSInstruments. The residual stress was determined using the Stoney's equation:

$$
\sigma=\frac{M_{s}}{6} \frac{h_{s}^{2}}{h_{f}}\left(\frac{1}{R}-\frac{1}{R_{0}}\right)
$$

where $h_{f}$ is the film thickness, $h_{s}$ is the substrate thickness $(200 \mu \mathrm{m}), M_{s}=180.3 \mathrm{GPa}$ is the biaxial modulus of the sub- strate, $R_{0}$ is the curvature radius of the substrate before the film deposition and $R$ is the curvature radius after the film deposition. These curvature radii were measured by White Light Interferometry using a Zygo NewView 7300 surface analyzer. The optical reflectivity measurements were performed in the 350-900 nm range using a Varian Cary 5000 UV-vis-NIR spectrophotometer with a integrating sphere coupled. Thus, the reflectance was calculated as $r=R_{S} / R_{t}$, where $R_{S}$ is the specular reflectance at normal incidence and $R_{t}$ is the total reflectance.

\section{Results and discussion}

\subsection{Nanocrystalline/amorphous self-phase separation}

In order to test the possibility to produce original microstructures around the reported transition between the amorphous and nanocrystalline phases of the out of equilibrium Zr-Mo system, thin films of thickness around $1500 \mathrm{~nm}$ were synthesized. Different values of the discharge current were applied to the Mo target, i.e $0.2,0.28$ and $0.4 \mathrm{~A}$, while keeping constant at $0.3 \mathrm{~A}$ the discharge current applied to the $\mathrm{Zr}$ target. It resulted in $\mathrm{Zr}$ Mo thin films containing 48, 60 and 67 at\% Mo, respectively. $\mathrm{X}$-ray diffractograms corresponding to these films are provided in Fig. 1(a). XRD data clearly indicate the possibility to modify the structure in the range of compositions explored. In more details, the signature of nanocrystalline bcc Mo is obtained for the highest Mo content (peaks are shifted with respect to pure Mo due to the incorporation of $\mathrm{Zr}$ atoms of larger atomic radius than Mo [22]), a wide diffraction signal corresponding to amorphous or ultrafine-grained phase is obtained for the lower Mo content and a mixture of both signals is obtained at the intermediate composition. We showed in [22] that the amorphous phase exhibits the same local order as the hcp solid solution of Mo into Zr. Hence, the constitutive phases obtained within the explored composition range are supersaturated nanocrystalline and amorphous solid solutions. This contrasts with the stable equilibrium phase diagram that contains (Mo) and ( $\mathrm{Zr}$ ) solid solutions of limited solubilities and one intermetallic C15 Laves Phase of $\mathrm{ZrMo}_{2}$ composition [27]. It is to be noted that the $\mathrm{ZrMo}_{2}$ Laves Phase was never observed in our $\mathrm{Zr-Mo}$ films neither using XRD nor using high resolution TEM. This is in line with the concept that the difficulty to form intermetallic phase of complex structure, due to high quenching rates associated to vapor condensation, favors the solid solution [28, 29]. The focus of this manuscript will be on the intermediate biphased composition, on the related manipulation of the surface topography and of the resulting optical reflectance.

In general, in absence of specific epitaxial orientation between the deposited phases and the substrate and without intentional thermal assistance, biphased coatings deposited using PVD methods consist of a matrix with nanodispersion of a secondary phase. Formation of such nanocomposites is driven both by thermodynamics and kinetics, and the very high cooling rates associated to the condensation of the gas phase using PVD methods play an important role in nanodispersing the secondary phase. In the present case a careful investigation of the film morphology reveals a different situation. In Fig. 1(c) is 

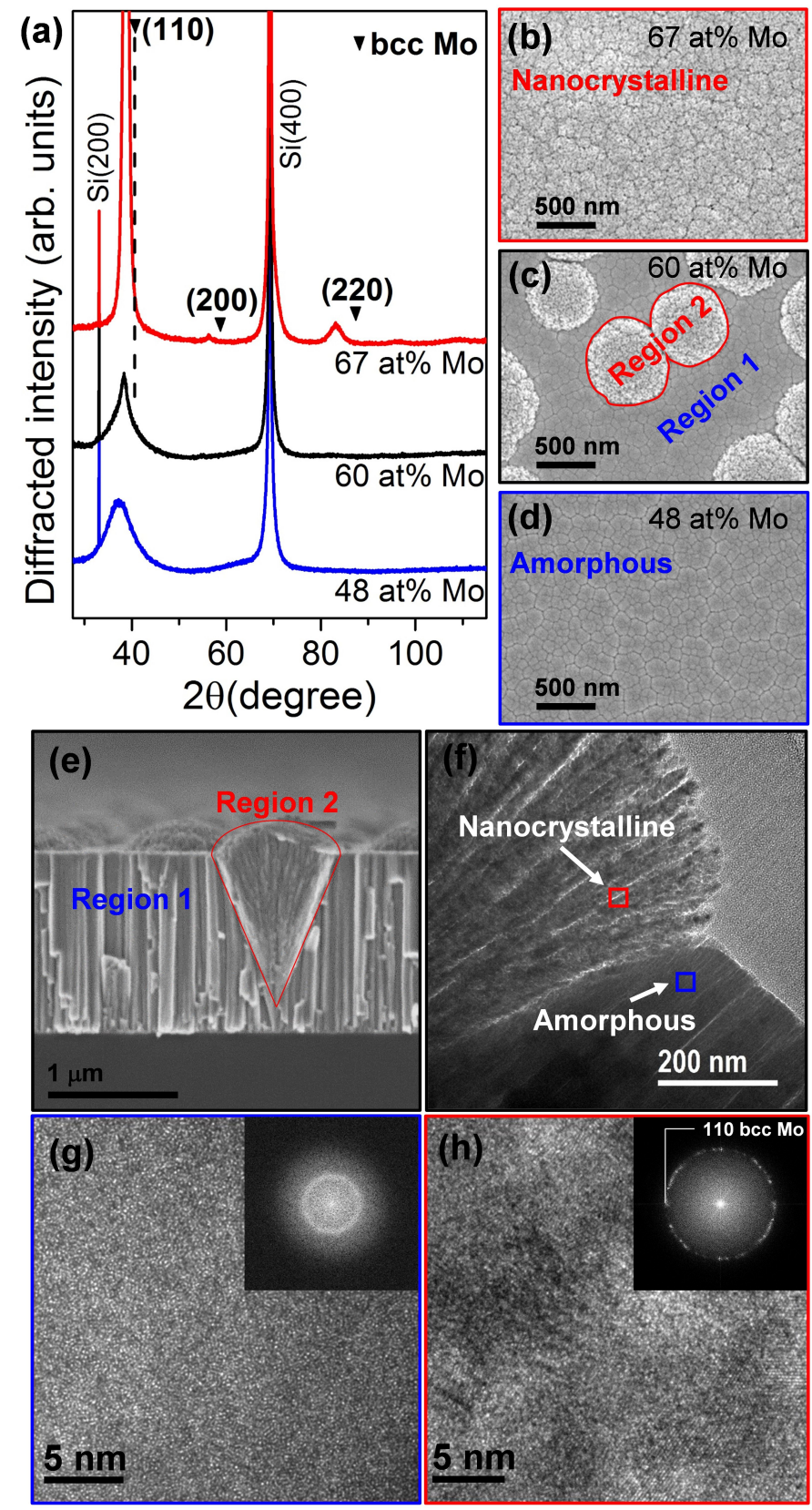

Figure 1: Morphology and structure of the films. (a) X-ray diffractograms of samples grown at different currents applied to the Mo target: blue line (0.2 A), black line $(0.28 \mathrm{~A})$ and red line $(0.4 \mathrm{~A})$. (b)-(d) Top-view SEM images corresponding to samples shown in (a). (e) SEM cross-sectional image of sample shown in (c). (f) TEM cross-sectional image of sample shown in (c). (g) TEM image and FFT from the region highlighted with a blue square in (f). (h) TEM image and FFT from the region highlighted with a red square in (f).

shown a SEM top-view image of the sample with $60 \%$ of Mo content. The image shows two regions with different morphologies: region 1 that is more flat and acts as a matrix embedding region 2 that is composed by zones of rounded shape. At the local scale the surface morphology of the region 1 is similar to that of the amorphous film of lower Mo content (see Fig. 1(d)), while the surface morphology of region 2 resembles that of the nanocrystalline film of higher Mo content (see Fig. 1(b)).

Fig. 1(e) shows the cross-sectional view of the biphased sam- ple and evidences that the shape of region 2 is conical inside the bulk of the film and terminates with a dome whose base is aligned with the surface of region 1. The TEM image of the film shown in Fig. 1(f) evidences the spatial separation of both regions. To bring further details on the local structure in both regions, high resolution TEM investigation and their FFT were performed. Fig. 1(g)-(h) show representative data collected in regions 1 and 2, marked by the blue and red squared in Fig. 1(f). The HRTEM image of Fig. 1(g) and diffuse halo in the corresponding FFT confirm the amorphous state in region 1. On the other hand, the HRTEM image of Fig. 1(h) confirms the nanocrystalline character of region 2 and the interplanar distance corresponding to the well-defined ring in the FFT pattern $(\mathrm{d}=0.233 \mathrm{~nm})$ matches with that extracted from XRD $(\mathrm{d}=0.234 \mathrm{~nm})$ for $(110)$ bcc Mo.

In summary, working at a composition between those of the amorphous and the nanocrystalline phases, we are able to separate these phases in well-defined spatial regions. Thus, the film grows initially amorphous and, for a certain thickness, the nanocrystalline phase nucleates and both phases continue growing together. Despite we report here on the self-separation phenomenon for a fixed composition, this effect is observed over a range of compositions. In the next section, the evolution of stress with thickness at the biphased composition will be discussed.

\subsection{Stress evolution with thickness}

In order to study how the morphology and the properties of the biphased composition evolve with the film thickness, several samples were grown under the same deposition conditions, but changing the deposition time. Fig. 2(a) shows the evolution of in-plane residual stress state of the film with thickness. From the figure, three different zones can be identified. Firstly, the compressive film stress decreases with increasing thickness in Zone 1, becoming almost zero, it then increases again through Zone 2, before levelling out (Zone 3). Fig. 2(b) shows the dependence with thickness of the coverage of the surface by the nanocrystalline phase, extracted from the analysis of SEM and AFM images. The inset in Fig. 2(b) shows the dependency of the stress with the surface coverage in the three zones already mentioned and typical top and cross-sectional SEM images from each zone are shown in Fig. 2(c). From Fig. 2(b) we can estimate to $162 \mathrm{~nm}$ the thickness at which the nucleation of the nanocrystalline phase starts, assuming that the coveragethickness dependence follows an exponential law. A schema of the morphology evolution with the thickness together with the stress in each zone is shown in Fig. 3(a). The stress evolution can be interpreted as follows:

Zone 1 (surface coverage below 15\%): In this zone, the nanocrystalline regions start to nucleate and grow without touching each other. As the thickness increases, the stress becomes less compressive, i.e. a tensile component of the stress develops due to the growth of the nanocrystalline regions. This can be understood taking in consideration that the grow of the crystalline phase can induce a volume contraction of the film [30, 31]. 
Zone 2 (surface coverage between $15 \%$ and $80 \%$ ): This zone is dominated by the coalescence of the nanocrystalline regions. A better look at the microstructure of the nano-crystalline regions shows that they are composed of nano-columns that grow from their axis in a feather-like morphology (see, for example, Fig. 1(f)). When two neighboring crystalline regions contact, the growth of the nano-columns in the in-plane direction is inhibited. Thus, the impingement of two nanocrystalline regions creates a repulsive force between them, as indicated by the arrows of Fig. 3(c). This repulsive force is the responsible of the increase in the compressive stress in zone 2.

Zone 3 (surface coverage above $80 \%$ ): Almost the entire surface is covered by the nanocrystalline phase. As a consequence, the driving mechanism for the compressive stress increase in zone 2 is interrupted and the stress stabilizes to a constant value.

\subsection{Possible path for the nanocrystalline/amorphous self- phase separation}

So far, the stress evolution has been explained from the moment when the nanocrystalline phase begins to nucleate (for thicknesses higher than approximately $162 \mathrm{~nm}$ ). For a thickness lower than $162 \mathrm{~nm}$, marked as a blue domain in Fig. 2(a) and Fig. 3(a), the whole film is amorphous and, consequently, the surface morphology is not markedly affected by a change of the film thickness. Nevertheless, the conditions for starting the nucleation of the nanocrystalline phase are defined in this zone. Because of this, an explanation of the possible path for the nucleation will be presented in the following.

First of all, we want to point out that the temperature measured at the substrate did not exceed $45{ }^{\circ} \mathrm{C}$, even after 3 hours of deposition. The nucleation of the nanocrystalline phase begins within the first 10 minutes of deposition during which the substrate temperature increase is negligible. Yet, the local temperature, particularly at the extreme surface is much higher and adatom diffusion can be sufficient to trigger crystal nucleation. Although we can not discard the possible influence of temperature evolution during deposition, we propose in the following how the local microstructure and chemical composition could initiate the nucleation of the nanocrystalline phase. Fig. 3(b) shows a typical TEM cross-sectional image of the films. It appears that the nucleation of the nanocrystalline phase starts at the grain boundary. Moreover, as was shown in the previous section, increasing the Mo content the structure of the films evolves from an amorphous to a nanocrystalline phase, with both phases coexisting at the biphased composition (see Fig. 1). This indicates that, besides the possibility to use the interfacial energy avaible at the grain boundary, the composition is an important factor to trigger the structural transition. Thus, at the biphased composition ( $60 \%$ of Mo content), the favoured phase in the initial growth stages is the amorphous one. Nevertheless, if by some mechanism the Mo content locally increases, a local transition to a crystalline structure can occur. This could therefore explain the transition observed when the film thickness reaches $162 \mathrm{~nm}$. To explain this, we take in consideration that the amorphous phase is growing in Volmer-Weber mode, as follows from its columnar structure (see Fig. 2(c)). In VolmerWeber type growth, in the post-coalescence regime, when the
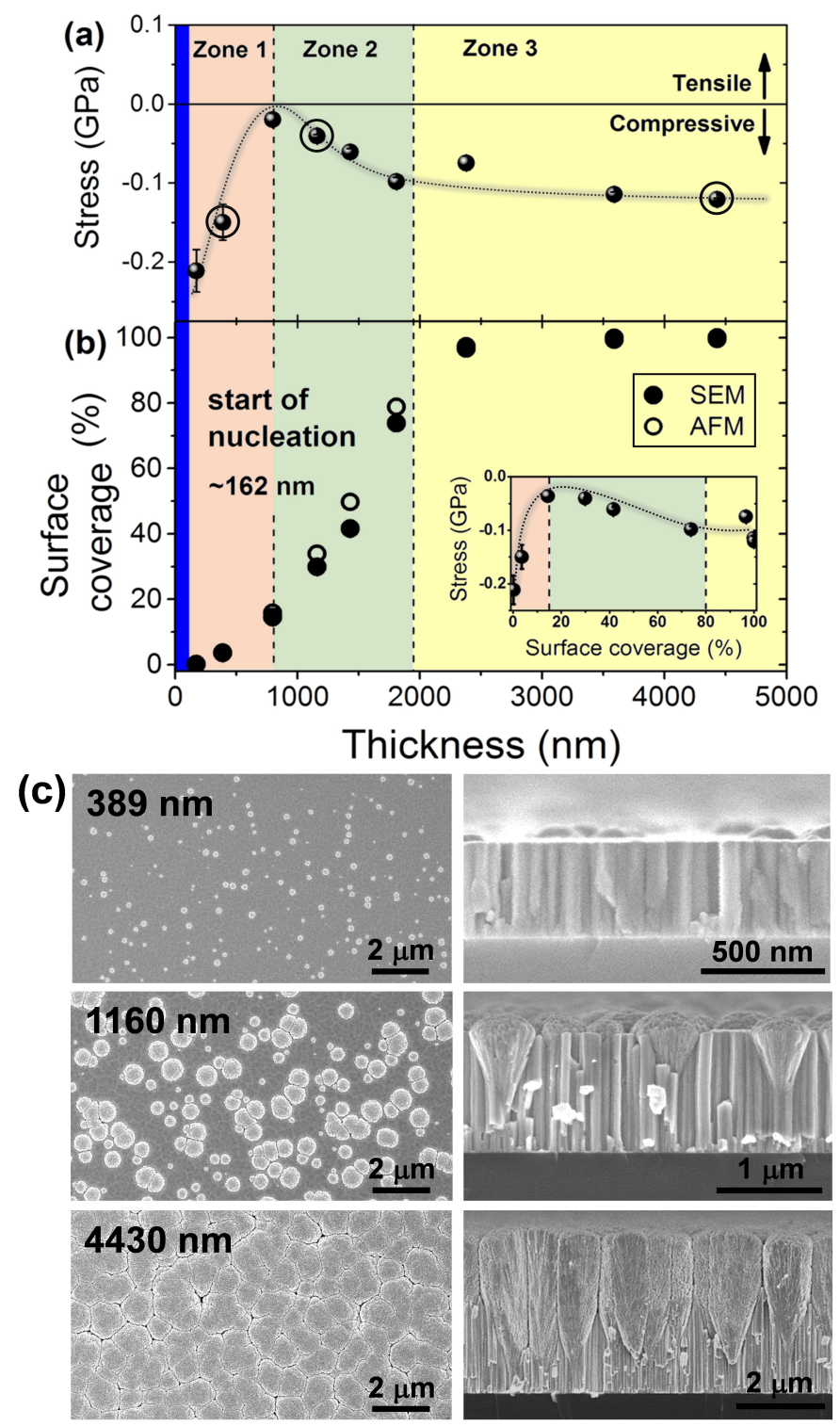

Figure 2: Evolution of residual stress (a) and surface coverage (b) with the increase of the films thickness at the biphased composition. The inset in (b) shows the dependency of stress with the surface coverage. Three different zones can be identified and top (left) and cross-sectional (right) SEM images representative of each zone are shown in (c).

grain boundaries between islands are created, it is possible to have a diffusion of adatoms from the surface of the islands to the grain boundaries, which depends of the adatom mobility $[32,33,34,35]$. This is due to the difference in the chemical potential between the growth surface and the grain boundaries. In most of the studies dedicated to understand the stress behavior in the post-coalescence regime, single element materials were taken in consideration and less informations exist about metallic alloy systems. Nevertheless, when two elements with different mobilities are diffusing into the grain boundaries, as in our case, their diffusion rates are generally different, provoking a preferential segregation of the most mobile element at the grain boundaries $[36,37]$. This means that, if the diffusion from 


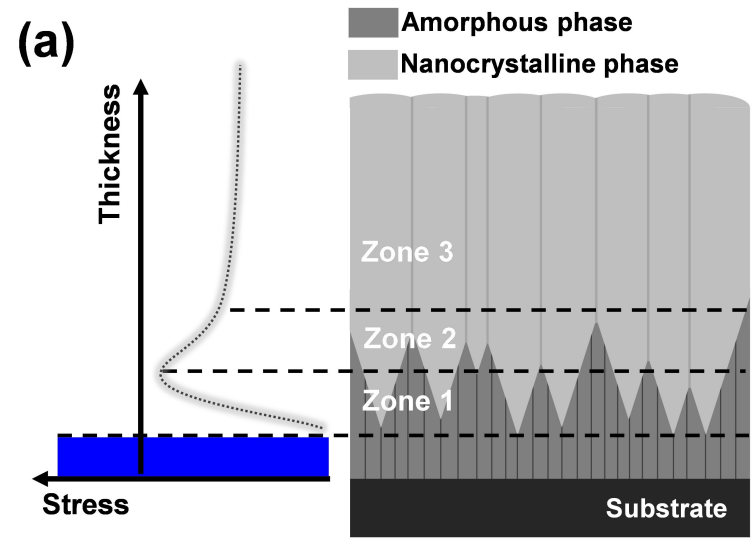

(b)

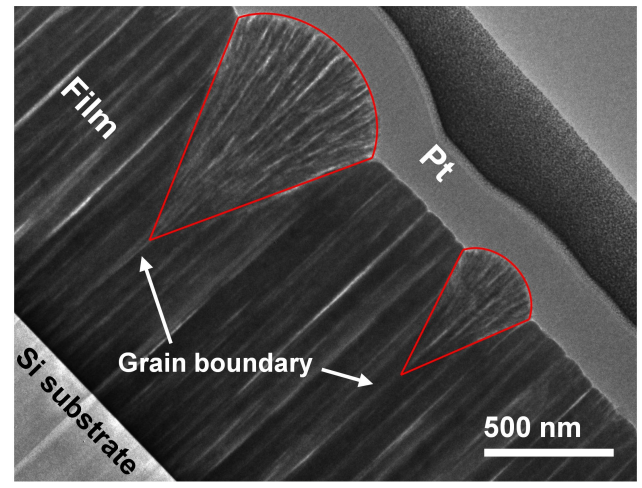

(c) Zone 2 : Crystalline regions coalescence

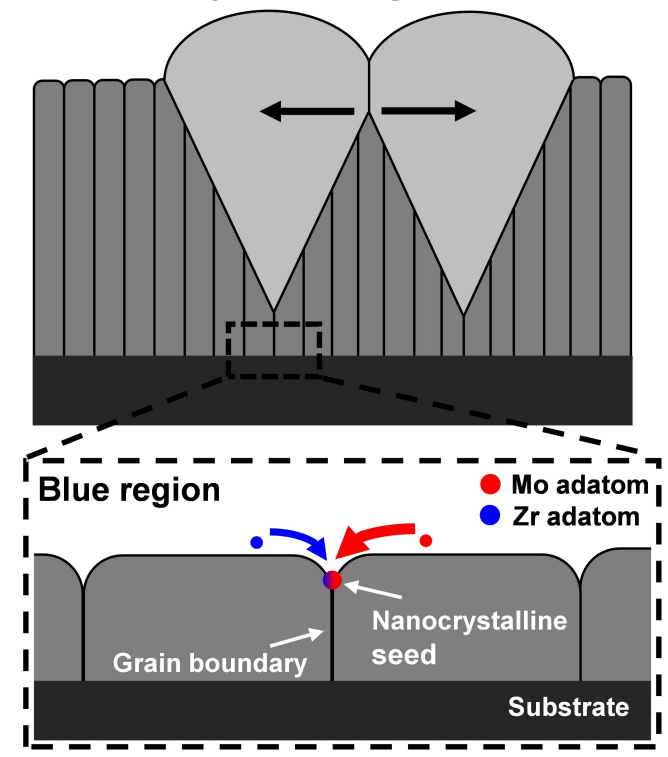

Figure 3: (a) Schematic showing the evolution with thickness of the film morphology and the stress. (b) TEM cross-sectional image of the film showing nanocrystalline regions growing from the grain boundary. (c) On top, the compressive stress generation by the impingement of two nanocrystalline regions is presented. The black arrows indicate the sense of the repulsive force that appear when two neighboring crystalline regions touch each others. At the bottom is shown the migration of the atoms from the surface to the grain boundary. The bigger red arrow comparing with the blue one means more Mo atoms going to the grain boundary. the surface into the grain boundary of the Mo adatoms is higher than that of the $\mathrm{Zr}$ adatoms, the $\mathrm{Mo} / \mathrm{Zr}$ proportion can be increased into the grain boundary, provoking the nanocrystalline phase nucleation. This process is illustrated at the bottom of Fig. 3(c), where a bigger red arrow indicates higher diffusion rate of Mo atoms into the grain boundary. Thereby, the difference in the diffusion rates of $\mathrm{Mo}$ and $\mathrm{Zr}$ into the grain boundaries can be the mechanism under the nanocrystalline phase nucleation. Following the Sigmund-Thompson theory for sputtering [38, 39], the most-likely kinetic energy of $\mathrm{Zr}$ and Mo sputtered particles is $E(\mathrm{Zr})=3.125 \mathrm{eV}$ and $E(\mathrm{Mo})=3.41 \mathrm{eV}$. The mean free path for collision of the same particle with the argon background gas atoms at $2 \mathrm{~Pa}$ is $\lambda_{f p}(\mathrm{Zr})=1.78 \mathrm{~cm}$ and $\lambda_{f p}(\mathrm{Mo})=2.52 \mathrm{~cm}$. Considering hard sphere collisions between the sputtered atoms and argon gas atoms, $\mathrm{Zr}$ and $\mathrm{Mo}$ atoms land on the film with $E_{f}(\mathrm{Zr})=0.217 \mathrm{eV}$ and $E_{f}(\mathrm{Mo})=$ $0.525 \mathrm{eV}$ most-likely kinetic energy [40]. Hence, more kinetic energy can be transformed into mobility for Mo adatoms and their diffusion to grain boundaries, supporting the proposed model.

As previously mentioned, the mobility is an important factor in order to understand the stress behaviour in the postcoalescence regime. The fact the highest value of the compressive stress was obtained at the beginning of zone 1 indicates that such a stress state developed during the growth of the initial amorphous phase (blue zone of Fig. 3(a)). This is an indication that the adatom mobility was high on this amorphous phase $[41,42]$. This seems contradictory with the high melting points of $\mathrm{Zr}\left(\mathrm{T}_{\mathrm{m}}=2128 \mathrm{~K}\right)$ and $\mathrm{Mo}\left(\mathrm{T}_{\mathrm{m}}=2896 \mathrm{~K}\right)$, and the fact that no thermal assistance was used during growth. Nevertheless, as recently shown [43], in the case of amorphous alloys, the temperature of reference is rather the glass transition temperature than the melting point. The glass transition temperature at the biphased composition calculated using the model presented in [44] is $\mathrm{T}_{\mathrm{g}}=997 \mathrm{~K}$, much lower than the melting temperatures of $\mathrm{Zr}$ and Mo. Hence, the amorphous structure favors the adatom mobility and, thereby, the diffusion of adatoms into the grain boundaries, likely facilitating the nucleation of the crystalline phase.

\subsection{Towards a generalization of the nanocrys- talline/amorphous competitive self-phase separation phenomenon}

So far, we have shown that a competitive self-separation of phases occurs in $\mathrm{Zr}-\mathrm{Mo}$ thin films obtained at 60 at\% of Mo, i.e. by working at a composition intermediate to those necessary to form single-phased amorphous and nanocrystalline films. Nevertheless, this is not something particular for the Zr-Mo system. For example, in sputter-deposited $\mathrm{Zr}-\mathrm{W}$ thin films, a transition from the amorphous to the crystalline phase was also observed [23]. This transition is illustrated in the X-ray diffractograms shown in Fig. 4 (a), in which the composition of $\mathrm{Zr}-\mathrm{W}$ thin films was varied by changing the discharge current applied to the $\mathrm{W}$ target while keeping constant the current applied to the $\mathrm{Zr}$ target. Fig. 4 (a) highlights that, as in the case of Zr-Mo, a mixture of both signals (the amorphous and the nanocrystalline) is obtained at the intermediate composition, in this case 72 at $\%$ of $\mathrm{W}$. 


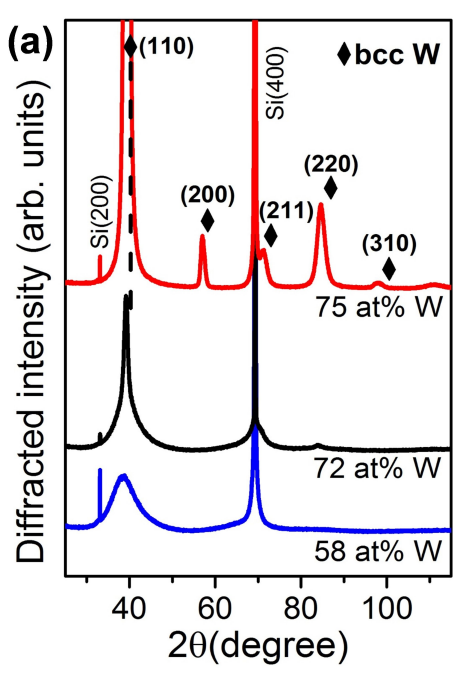

(b)

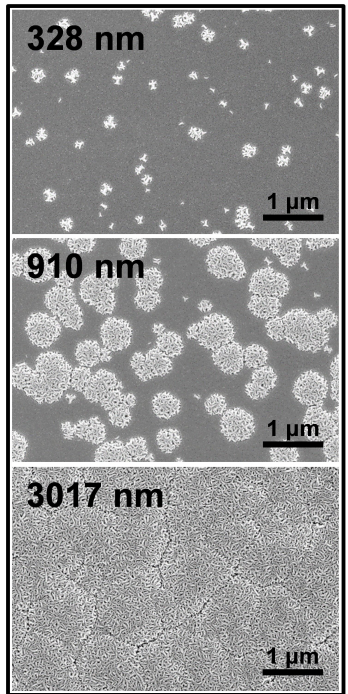

(c)

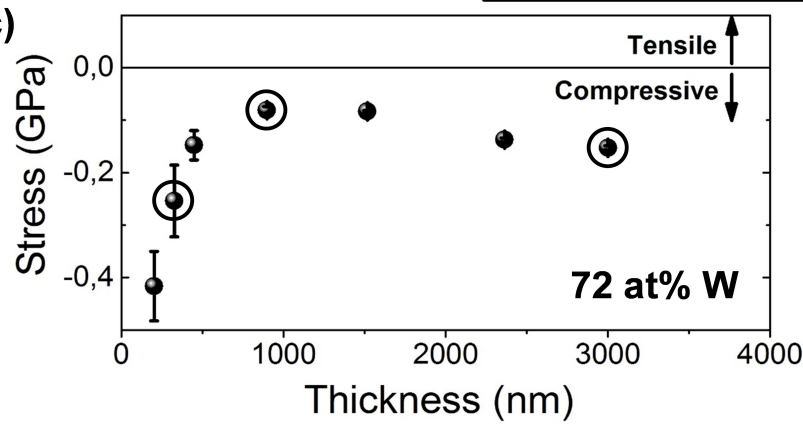

Figure 4: Sputter-deposited Zr-W thin films. (a) X-ray diffractograms for films grown with different at $\%$ of $\mathrm{W}$ content. (b) Top SEM images at $\mathrm{W}$ content of 72 at\% for different film thickness. (c) Evolution of residual stress with the increase of the films thickness at 72 at\% of $\mathrm{W}$ content.

On the other hand, SEM top-views of samples obtained at this composition but with different thicknesses are shown in Fig. 4 (b), revealing the competitive separation of the amorphous and the nanocrystalline phases. The SEM top-views also show the increase of the nanocrystalline/amorphous ratio with the sample thickness. The stress-thickness dependency displayed in Fig. 4 (c) shows a similar behavior to that presented in section 3.2 for Zr-Mo (see Fig. 2 (a)), meaning that the growth mechanisms are similar in both systems. Hence, competitive self-separation is not restricted to the $\mathrm{Zr}$-Mo system and is likely general to other binary alloys exhibiting a composition-driven transition from an amorphous to a nanocrystalline phases. A similar separation has also been reported in the case of AlN [45] and $\mathrm{TiO}_{2}$ [14] films.

As an example to support such a possible generalization of the process, we focus in the following on the common characteristics of phase separation phenomenon reported in $\mathrm{TiO}_{2}$ films [14] with that occurring in the Zr-Mo system. These films show a transition from amorphous state with local anatase character to nanocrystalline rutile, the controlling parameter being the oxygen partial pressure during film growth that sets the oxygen content in the Ti-O films. Similar to the results reported here, in biphased Ti-O films, growth initiates with amorphous
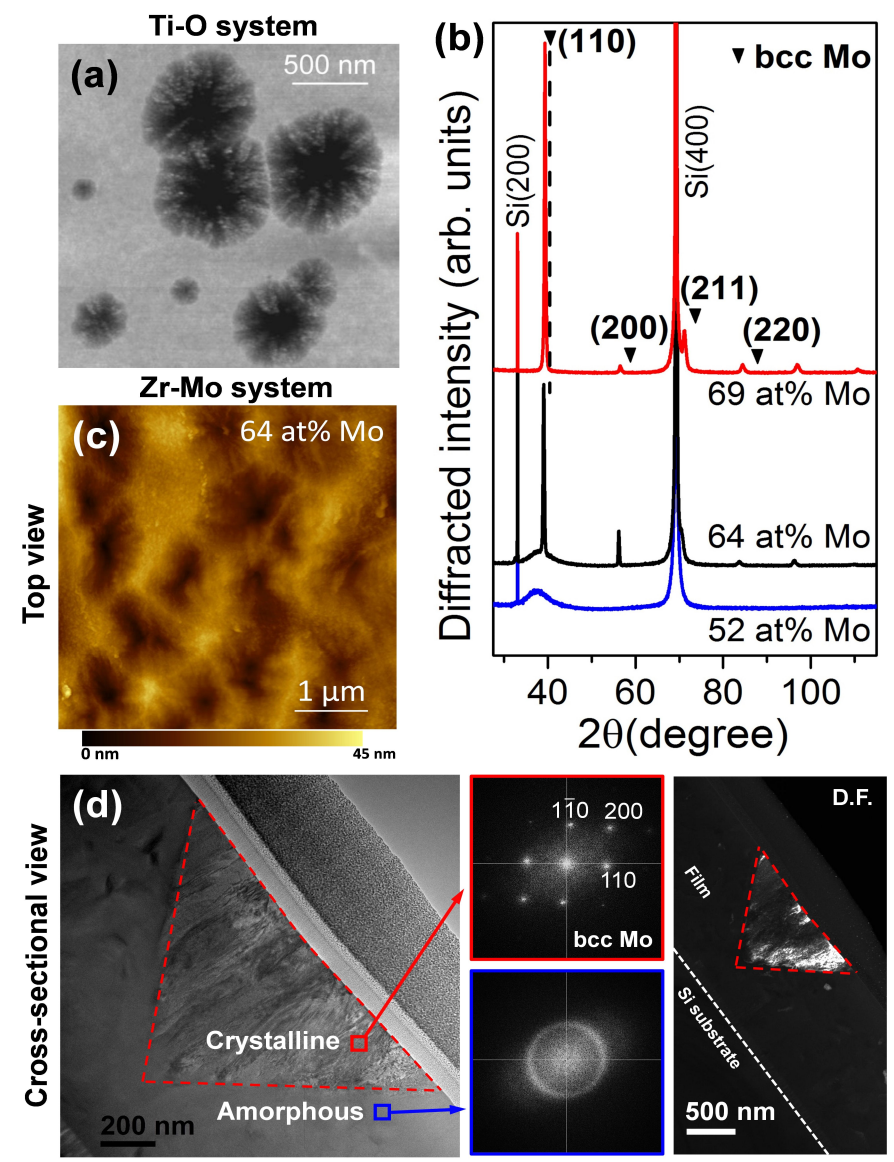

Figure 5: Films grown at low working pressures. (a) AFM image of a $\mathrm{TiO}_{2}$ film grown at $0.3 \mathrm{~Pa}$. Lower heights appear in dark (figure reproduced with permission from ref. [14]). (b) X-ray diffractograms of Zr-Mo thin films deposited at $0.5 \mathrm{~Pa}$. (c) Top-view AFM image of sample with 64 at\% of Mo content. (d) TEM cross-sectional analysis of sample with 64 at\% of Mo content. TEM image (left panel), FFT from the regions highlighted in the TEM image (middle panels), and dark field image (right panel) of the film.

phase before the nanocrystalline phase nucleates. The difference between the results obtained with $\mathrm{Zr}$-Mo films and reported in section 3.2 and those for the $\mathrm{TiO}_{2}$ films is the geometrical shape of the nanocrystalline phases. In the case of $\mathrm{Zr}$ Mo, the nanocrystalline phase grows with a dome-like shape on the surface (see Fig. 2(c)), while $\mathrm{TiO}_{2}$ films reported in [14] exhibit hollow structures (holes) on the surface (see Fig. 5(a)). Such difference can be understood in terms of the growth rates of the nanocrystalline phase in the in-plane (parallel to the film surface) and vertical (perpendicular to the film surface) directions. An increase of the growth rate in the in-plane direction with reference to the vertical can lead to diffusion of adatoms from the center of nanocrystalline precipitates towards their periphery. This can be supported by an improved diffusion of adatoms. In order to test this hypothesis we have deposited Zr-Mo films at a lower pressure of $0.5 \mathrm{~Pa}$. A lower working pressure increases the mean free path and decreases the number of collisions a sputtered atom experiences before it lands on the film surface. This results in the increase of the kinetic energy of the arriving adatoms, favoring the mobility in the in-plane 
direction and, in consequence, the growth rate in this direction increases. The deposition parameters were set such that the deposition rates for $0.5 \mathrm{~Pa}(28 \mathrm{~nm} / \mathrm{min})$ and $2 \mathrm{~Pa}(24 \mathrm{~nm} / \mathrm{min})$ were very close. Hence, we can discard a major contribution of the deposition rate on the morphology of the crystalline regions grown using the two deposition pressures. Fig. 5 (b) shows $\mathrm{X}$-ray diffractograms of samples obtained at different values of the current applied to the Mo target $(0.2,0.3$ and $0.4 \mathrm{~A})$ while keeping constant $(0.3 \mathrm{~A})$ the current applied to the $\mathrm{Zr}$ target. As in the case of $\mathrm{Zr}$-Mo films obtained at $2 \mathrm{~Pa}$, a transition from an amorphous to a crystalline structure occurs. At the intermediate composition (64 at\% of Mo in this case) there is a mixture of both signals. The AFM image shown in Fig. 5 (c) for sample obtained at 64 at $\%$ of Mo reveals a completely different surface morphology compared with that of biphased samples obtained at $2 \mathrm{~Pa}$. In this case, the sample shows hollow structures on the surface that are similar to those obtained for $\mathrm{TiO}_{2}$ samples (see Fig. 5 (a)). In addition, cross-sectional TEM analysis performed to this sample confirms the crystalline and amorphous character of the hollows and the surrounding region, respectively (see middle panels in Fig. 5 (d)). Also, the geometrical shape of the hollows in the case of Zr-Mo system (see TEM and dark field images in the left and right panels of Fig. 5 (d)) is similar to that presented in $\mathrm{TiO}_{2}$ samples [14], proving that the phase separation phenomenon is the same in both systems but the geometrical shape of the nanocrystalline region is affected by the deposition pressure.

\subsection{Thickness-modulated reflectance}

The possibility to modulate the surface morphology at the biphased composition by changing the film thickness can be exploited to control the surface-related properties. For example, the wettability of a solid surface is dominated by both its surface free energy and its geometric structure (or surface roughness) $[46,47]$. Therefore, by selecting an adequate system (in which the competitive self-phase separation phenomenon occurs) and controlling the film thickness, both the free energy and the roughness of the surface can be modified. Hence, the surface wettability could be controlled. Another possible route to functionalize films that exhibit the competitive self-phase separation phenomenon is the ability to control the size and number of "grains" formed by the nanocrystalline regions. Thus, by changing the film thickness, the number of contact points with a reciprocating surface can be changed and, consequently, the friction could be controlled.

Here we propose to illustrate the potential of the competitive self-separation of amorphous and nanocrystalline phases by probing its ability to modulate the optical reflectance. Due to the increase of the nanocrystalline/amorphous ratio with thickness, a change in the surface roughness is expected. This is pointed out in Fig. 6, where the dependence of the surface roughness (black circles) with the thickness obtained from AFM measurements is shown. A smooth variation of the roughness from 5 to $100 \mathrm{~nm}$ is achieved by increasing the thickness up to $2 \mu \mathrm{m}$. Above $2 \mu \mathrm{m}$, the decrease of the roughness with thickness indicates a smoothening of the surface after it is completely covered by the nanocrystalline phase (zone 3 in Fig. 2).
The bottom panels of Fig. 6 show the AFM images (left) for some of the samples obtained with their corresponding height distribution functions (right). The first peak of the height distribution functions is representative of the background, which in this case belongs to the height distribution function of the amorphous region. On the other hand, the second peak, coming from higher structures, represents the height distribution function of the dome-like nanocrystalline region.

The possibility to control the roughness can be used to modulate the optical properties of the films. Fig. 7 shows the reflectance spectra for the different thicknesses obtained. The correlation between the surface roughness and reflectance is evidenced in Fig. 6, where the dependence of the reflectance with thickness at a wavelength of $550 \mathrm{~nm}$ is shown. Therefore, by changing the film thickness, and with that the surface roughness, we are able to control the reflectance at a certain wavelength.

Another important point to be addressed comes from the analysis of the zooms shown in Fig. 7 for film thicknesses of 1160,1430 and $1810 \mathrm{~nm}$. A minimum is observed in the reflectance spectra. Furthermore, the position of this minimum is changing with film thickness. Moreover, it is evident from the spectrum of the $1810 \mathrm{~nm}$ film that reflectance oscillates, as in an interference phenomenon. This can be understood by taking into account the height distribution functions of these samples, which are shown in Fig. 6. As said before, the distributions show two predominant heights corresponding to the amorphous (background) and nanocrystalline (dome-like) regions. Therefore, if a light wave approaches the film, the presence of two levels of height creates a path difference, which results in the creation of an interference pattern. The condition for minimum intensity in an interference pattern is:

$$
\Delta=(n+1 / 2) \lambda_{\min },
$$

where $\Delta$ is the path difference, $n$ is an integer value and $\lambda_{\text {min }}$ is the wavelength at which a minimum in intensity is detected. Thereby, for the first minimum of interference $n=0$ and taking into account that the path difference for a wave coming perpendicular to the films surface is $\Delta=2 h$, where $h$ is the distance between the two predominant heights, we can rewrite eq. (2) as:

$$
h=\frac{\lambda_{\min }}{4} .
$$

Eq. (3) gives us the height difference $h$ that two height levels must have to cause a minimum in the reflectance spectrum at a wavelength $\lambda_{\min }$. Taking into account the values of $\lambda_{\min }$ extracted from the reflectance spectra (see Fig. 7), the values of $h$ were calculated and the results are shown in Fig.6. In this way, the higher height reached by the nanocrystalline phase with respect to the amorphous one creates a difference in height levels that is responsible for the minimum and maximum in the reflectance intensity. Thus, as the dome-like nanocrystalline phase grows with the increase of the film thickness, the difference in height levels also increases, causing a shift of the minimum of interference to longer wavelengths. 

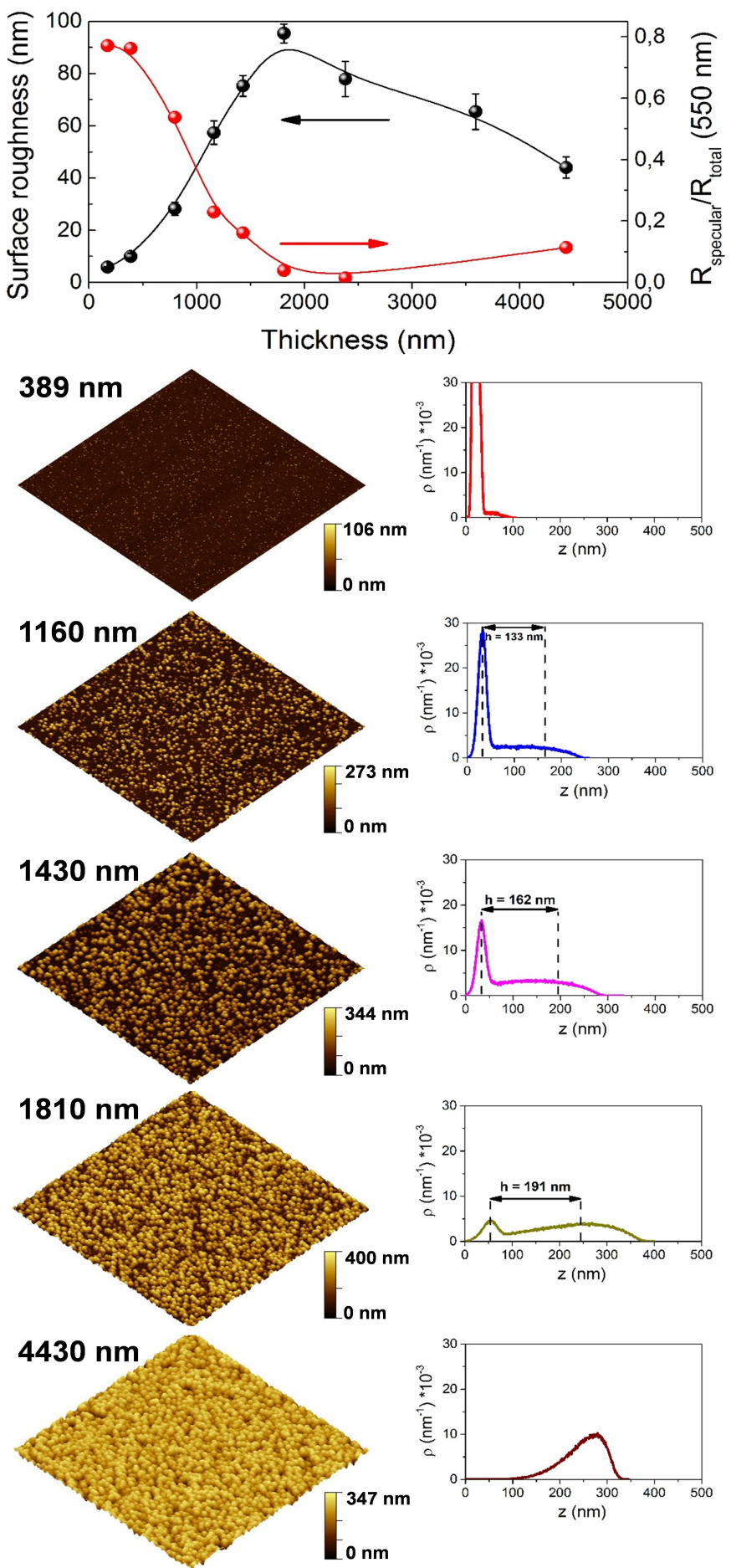

Figure 6: Evolution with thickness of the surface roughness (black circles) and the reflectance at a wavelength of $550 \mathrm{~nm}$ (red circles). The bottom panels show $45 \times 45 \mu \mathrm{m}^{2}$ AFM images (left) for some of the samples obtained with their corresponding height distribution functions (right). The values of $h$ for film thicknesses of 1160,1430 and $1810 \mathrm{~nm}$ were calculated using eq. (3).

It is important to note that from eq. (3) it follows that in order to see the first minimum of the interference pattern in the range of wavelength studied (between 350 and $900 \mathrm{~nm}$ ), the height difference of the two level of heights have to be between 87.5

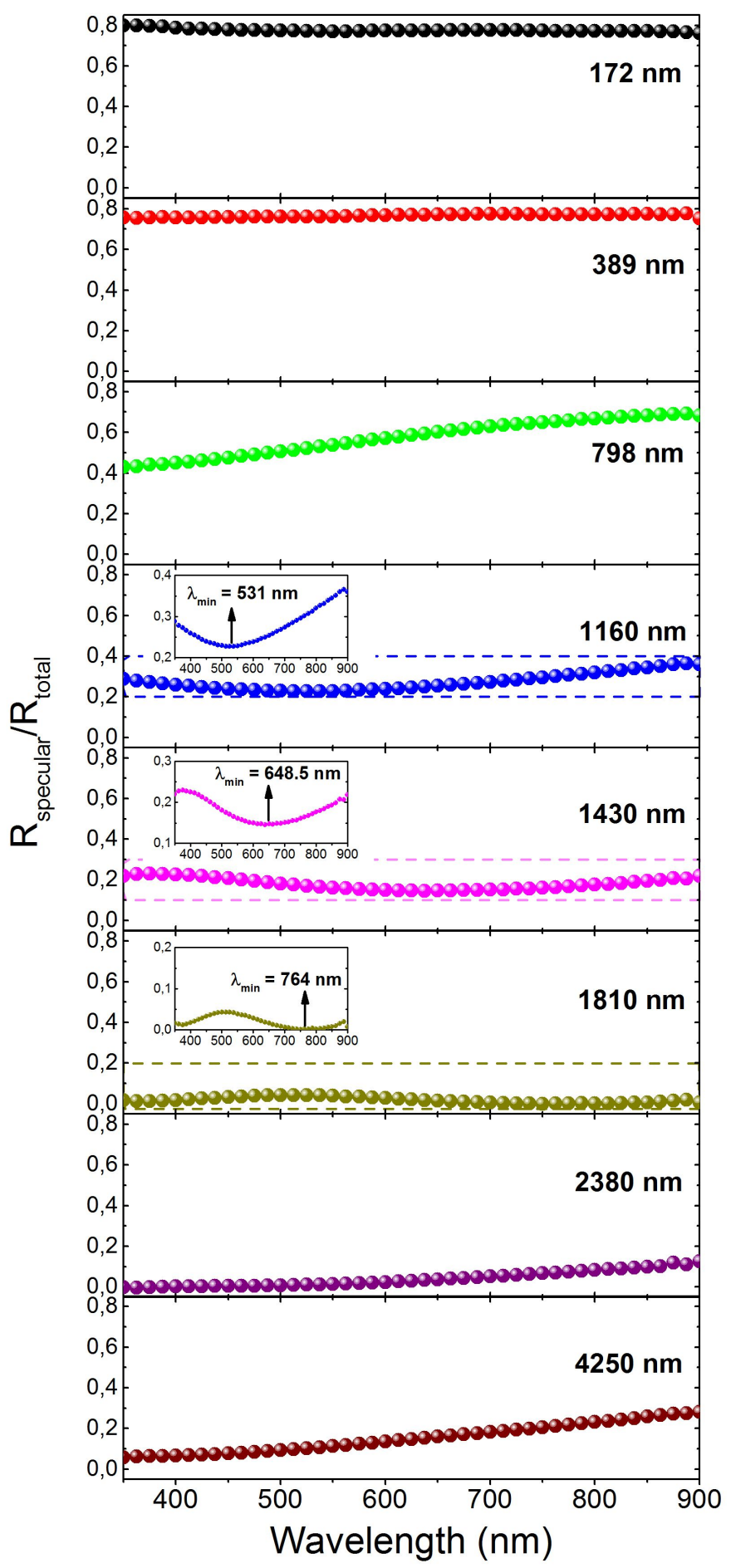

Figure 7: Reflectance spectra as the thickness increases. The zooms for film thicknesses of 1160, 1430 and $1810 \mathrm{~nm}$ shown a minimum in the reflectance spectra.

and $225 \mathrm{~nm}$. This is the case for the samples with a thickness between 1160 and $1810 \mathrm{~nm}$ while for the others thicknesses this condition is not satisfied. This is the reason why, for other thickness values, a minimum is not observed in the reflectance spectrum in the range of wavelengths studied. On the other 
hand, it is also important to have a similar proportion between the lowest and the highest level, that is, the surface coverage should be around $50 \%$. This condition is better fulfilled when the samples thickness is between 1160 and $1810 \mathrm{~nm}$, where the surface coverage varies between 30 and $80 \%$.

Even for thicknesses between 1160 and $1810 \mathrm{~nm}$, where the samples satisfy the conditions explained above, there is not a strong difference between the minimum and the maximum of the reflectance, i.e. the interference pattern is not so well defined. This is because we do not have two well-defined levels of height. On the contrary, there is a dispersion of the height values fundamentally within the height distribution of the nanocrystalline region, as can be follow from Fig. 6. Thus, in order to obtain a better defined interference pattern, a reduction in the dispersion of the height values is necessary. This last issue can be addressed by changing the deposition conditions, for example, the deposition pressure. In this way, the geometry of the nanocrystalline region could be changed and obtaining, for example, flatter structures would decrease the dispersion in the height distribution.

Thickness and/or composition-dependent reflectivity control opens an avenue for development of local manipulation of optical properties at micron scale. We foresee an interest in the field of micro-devices.

\section{Conclusions}

In summary, we have shown that by working at a composition between those necessary to obtain the amorphous and the nanocrystalline phases in sputter-deposited Zr-Mo thin films, a competitive self-separation of these phases in well defined spatial regions can be achieved. Due to the particular geometry in which the nanocrystalline phase grows, the morphology at the biphased composition changes with the film thickness. The influence of the morphology evolution on the stress was discussed. Moreover, a possible mechanism for the selfseparation of phases was presented. We show that the selfseparation of phases as presented here is a more general phenomenon. It can be obtained in other binary alloys showing a composition-driven transition from an amorphous to a crystalline phase. Thus, by changing the parameter that triggers this transition, i.e. the $\mathrm{Mo}(\mathrm{W})$ content in the case of $\mathrm{Zr}-\mathrm{Mo}(\mathrm{W})$ alloys and the oxygen partial pressure in the Ti-O system, the self-separation of phases can be obtained. Moreover, the geometry in which the crystalline phase grows can be changed by varying the working pressure. Finally, we show that because of the competitive self-phase separation, it is possible to control the surface roughness of the films and, thereby, to tune the reflectance at a determined wavelength, using the film thickness as a control parameter. We also show that the presence of a minimum in the reflectance spectra is due to the presence of two height levels in the height distribution function. The present findings opens new possibilities to manipulate the optical reflectivity at local scale.

\section{Acknowledgments}

The "Université franco-allemande" (UFA) and the "Ministère de l'Enseignement Supérieur et de la Recherche" are deeply acknowledged for the PhD scholarship of Alejandro Borroto within the PhD-track in Materials Science and Engineering at UFA. The Da $\mu$ m competence center of IJL is deeply acknowledged for access to UHV magnetron sputtering deposition facility.

\section{References}

[1] M. Geissler, Y. Xia, Patterning: Principles and some new developments, Adv. Mater. 16 (2004) 1249-1269.

[2] A. F. Lasagni, Laser interference patterning methods: Possibilities for high-throughput fabrication of periodic surface patterns, Adv. Opt. Technol. 6 (2017) 265-275.

[3] E. Hutli, M. Nedeljkovic, A. Bonyár, Controlled modification of the surface morphology and roughness of stainless steel 316 by a high speed submerged cavitating water jet, Appl. Surf. Sci. 458 (2018) 293-304.

[4] W. Z. Qiu, H. C. Yang, Z. K. Xu, Dopamine-assisted co-deposition: An emerging and promising strategy for surface modification, Adv. Colloid Interface Sci. 256 (2018) 111-125.

[5] M. Ma, R. M. Hill, Superhydrophobic surfaces, Curr. Opin. Colloid Interface Sci. 11 (2006) 193-202.

[6] R. Estevam-Alves, D. Günther, S. Dani, S. Eckhardt, T. Roch, C. R. Mendonca, I. N. Cestari, A. F. Lasagni, UV direct laser interference patterning of polyurethane substrates as tool for tuning its surface wettability, Appl. Surf. Sci. 374 (2016) 222-228.

[7] A. Bekmurzayeva, W. J. Duncanson, H. S. Azevedo, D. Kanayeva, Surface modification of stainless steel for biomedical applications: Revisiting a century-old material, Mater. Sci. Eng. C 93 (2018) 1073-1089.

[8] L. Müller-Meskamp, Y. H. Kim, T. Roch, S. Hofmann, R. Scholz, S. Eckardt, K. Leo, A. F. Lasagni, Efficiency enhancement of organic solar cells by fabricating periodic surface textures using direct laser interference patterning, Adv. Mater. 24 (2012) 906-910.

[9] W. Chamorro, J. Ghanbaja, Y. Battie, A. En Naciri, F. Soldera, F. Mücklich, D. Horwat, Local structure-driven localized surface plasmon absorption and enhanced photoluminescence in $\mathrm{ZnO}-\mathrm{Au}$ thin films, J. Phys. Chem. C 120 (2016) 29405-29413.

[10] H. Rinnert, M. Vergnat, A. Burneau, Evidence of light-emitting amorphous silicon clusters confined in a silicon oxide matrix, J. Appl. Phys. 89 (2001) 237-243.

[11] N. Ghafoor, L. S. Johnson, D. O. Klenov, J. Demeulemeester, P. Desjardins, I. Petrov, L. Hultman, M. Odén, Nanolabyrinthine ZrAlN thin films by self-organization of interwoven single-crystal cubic and hexagonal phases, APL Mater. 1 (2013) 022105.

[12] Y. Wang, J. Ghanbaja, S. Bruyère, F. Soldera, D. Horwat, F. Mücklich, J. F. Pierson, Room temperature self-assembled growth of vertically aligned columnar copper oxide nanocomposite thin films on unmatched substrates, Sci. Rep. 7 (2017) 11122.

[13] J. L. MacManus-Driscoll, P. Zerrer, H. Wang, H. Yang, J. Yoon, A. Fouchet, R. Yu, M. G. Blamire, Q. Jia, Strain control and spontaneous phase ordering in vertical nanocomposite heteroepitaxial thin films, Nature Mater. 7 (2008) 314-320.

[14] R. Gago, M. Vinnichenko, A. Redondo-Cubero, Z. Czigány, L. Vázquez, Surface morphology of heterogeneous nanocrystalline rutile/amorphous anatase $\mathrm{TiO}_{2}$ films grown by reactive pulsed magnetron sputtering, Plasma Process. Polym. 7 (2010) 813-823.

[15] J. F. Pierson, M. Baija, D. Horwat, Silver islands formed after air annealing of amorphous AgCuMnO sputtered films, J. Cryst. Growth 311 (2009) 349-354.

[16] Suyalatu, N. Nomura, K. Oya, Y. Tanaka, R. Kondo, H. Doi, Y. Tsutsumi, T. Hanawa, Microstructure and magnetic susceptibility of as-cast $\mathrm{Zr}-\mathrm{Mo}$ alloys, Acta Biomater. 6 (2010) 1033-1038.

[17] Suyalatu, R. Kondo, Y. Tsutsumi, H. Doi, N. Nomura, T. Hanawa, Effects of phase constitution on magnetic susceptibility and mechanical properties of Zr-rich Zr-Mo alloys, Acta Biomater. 7 (2011) 4259-4266. 
[18] F. Y. Zhou, B. L. Wang, K. J. Qiu, L. Li, J. P. Lin, H. F. Li, Y. F. Zheng, Microstructure, mechanical property, corrosion behavior, and in vitro biocompatibility of Zr-Mo alloys, J. Biomed. Mater. Res. Part B 101B (2013) 237-246.

[19] F. Y. Zhou, K. J. Qiu, H. F. Li, T. Huang, B. L. Wang, L. Li, Y. F. Zheng, Screening on binary $\mathrm{Zr}-1 \mathrm{Mo}(\mathrm{X}=\mathrm{Ti}, \mathrm{Nb}, \mathrm{Mo}, \mathrm{Cu}, \mathrm{Au}, \mathrm{Pd}, \mathrm{Ag}, \mathrm{Ru}, \mathrm{Hf}$ and $\mathrm{Bi}$ ) alloys with good in vitro cytocompatibility and magnetic resonance imaging compatibility, Acta Biomater. 9 (2013) 9578-9587.

[20] X. Sun, W. Zhou, K. Kikuchi, N. Nomura, A. Kawasaki, H. Doi, Y. Tsutsumi, T. Hanawa, Fabrication and characterization of a low magnetic $\mathrm{Zr}$ 1Mo alloy by powder bed fusion using a fiber laser, Metals 7 (2017) 501.

[21] P. Y. Park, E. Akiyama, H. Habazaki, A. Kawashima, K. Asami, K. Hashimoto, The corrosion behavior of sputter-deposited amorphous Mo-Zr alloys in $12 \mathrm{M} \mathrm{HCl}$, Corros. Sci. 37 (1995) 307-320.

[22] A. Borroto, S. Bruyère, N. Thurieau, C. Gendarme, E. Jimenez-Pique, J. J. Roa, J. F. Pierson, F. Mücklich, D. Horwat, Structural and mechanical properties of $\mathrm{Zr}_{1-\mathrm{x}} \mathrm{Mo}_{\mathrm{x}}$ thin films: From the nano-crystalline to the amorphous state, J. Alloys Compd. 729 (2017) 137-143.

[23] D. Horwat, M. Dehmas, E. Aubry, J. Zollinger, S. Migot, J. F. Pierson, Properties of nanocrystalline and nanocomposite $\mathrm{W}_{\mathrm{x}} \mathrm{Zr}_{1-\mathrm{x}}$ thin films deposited by co-sputtering, Intermetallics 17 (2009) 421-426.

[24] H. Davies, The reflection of electromagnetic waves from a rough surface, Proc. IEE. Pt. III 101 (1954) 209-214.

[25] H. E. Bennett, J. O. Porteus, Relation between surface roughness and specular reflectance at normal incidence, J. Opt. Soc. Am. 51 (1961) 123129.

[26] J. E. Harvey, S. Schröder, N. Choi, A. Duparré, Total integrated scatter from surfaces with arbitrary roughness, correlation widths, and incident angles, Opt. Eng. 51 (2012) 013402.

[27] R. J. Pérez, B. Sundman, Thermodynamic assessment of the Mo-Zr binary phase diagram, Calphad 27 (2003) 253-262.

[28] J. M. Lopez, J. A. Alonso, L. J. Gallego, Determination of the glassforming concentration range in binary alloys from a semiempirical theory: Application to Zr-based alloys, Phys. Rev. B 36 (1987) 3716-3722.

[29] S. Zhao, J. H. Li, S. M. An, S. N. Li, B. X. Liu, Atomistic modeling to investigate the favored composition for metallic glass formation in the CaMgNi ternary system, Phys. Chem. Chem. Phys. 19 (2017) 12056.

[30] B. Krause, G. Abadias, A. Michel, P. Wochner, S. Ibrahimkutty, T. Baumbach, Direct observation of the thickness-induced crystallization and stress build-up during sputter-deposition of nanoscale silicide films, ACS Appl. Mater. Interfaces 8 (2016) 34888-34895.

[31] A. Fillon, G. Abadias, A. Michel, C. Jaouen, P. Villechaise, Influence of phase transformation on stress evolution during growth of metal thin films on silicon, Phys. Rev. Lett. 104 (2010) 096101.

[32] E. Chason, B. W. Sheldon, L. B. Freund, J. A. Floro, S. J. Hearne, Origin of compressive residual stress in polycrystalline thin films, Phys. Rev. Lett. 88 (2002) 156103.

[33] E. Chason, A kinetic analysis of residual stress evolution in polycrystalline thin films, Thin Solid Films 526 (2012) 1-14.

[34] E. Chason, J. W. Shin, S. J. Hearne, L. B. Freund, Kinetic model for dependence of thin film stress on growth rate, temperature, and microstructure, J. Appl. Phys. 111 (2012) 083520.

[35] A. M. Engwall, Z. Rao, E. Chason, Origins of residual stress in thin films: Interaction between microstructure and growth kinetics, Mater. Des. 110 (2016) 616-623.

[36] B. Fu, G. B. Thompson, Compositional dependent thin film stress states, J. Appl. Phys. 108 (2010) 043506.

[37] T. M. Kaub, P. Felfer, J. M. Cairney, G. B. Thompson, Influence of Ni solute segregation on the intrinsic growth stresses in $\mathrm{Cu}(\mathrm{Ni})$ thin films, Scr. Mater. 113 (2016) 131-134.

[38] P. Sigmund, Theory of sputtering. i. sputtering yield of amorphous and polycrystalline targets, Phys. Rev. 184 (1969) 383.

[39] M. W. Thompson, The energy spectrum of ejected atoms during the high energy sputtering of gold, Philos. Mag. 18 (1968) 377.

[40] P. Brault, E. C. Neyts, Molecular dynamics simulations of supported metal nanocatalyst formation by plasma sputtering, Catal. Today 256 (2015) 3-12.

[41] W. D. Nix, B. M. Clemens, Crystallite coalescence: A mechanism for intrinsic tensile stresses in thin films, J. Mater. Res. 14 (1999) 3467-3473.

[42] R. C. Cammarata, T. M. Trimble, D. J. Srolovitz, Surface stress model for intrinsic stresses in thin films, J. Mater. Res. 15 (2000) 2468-2474.
[43] D. Depla, B. R. Braeckman, Quantitative correlation between intrinsic stress and microstructure of thin films, Thin Solid Films 604 (2016) 9093.

[44] Z. Lu, J. Li, Correlation between average melting temperature and glass transition temperature in metallic glasses, Appl. Phys. Lett. 94 (2009) 061913.

[45] V. Brien, P. Miska, B. Bolle, P. Pigeat, Columnar growth of AlN by r.f. magnetron sputtering: Role of the $\{10 \overline{1} 3\}$ planes, J. Crystal Growth 307 (2007) 245-252.

[46] X. Feng, L. Jiang, Design and creation of superwetting/antiwetting surfaces, Adv. Mater. 18 (2006) 3063-3078.

[47] E. Ueda, P. A. Levkin, Emerging applications of superhydrophilicsuperhydrophobic micropatterns, Adv. Mater. 25 (2013) 1234-1247. 